\title{
Vacuum leak search on the Wendelstein 7-X cryostat vessel
}

\author{
J. Baldzuhn ${ }^{*}$, H. Reimer ${ }^{1}$, C. Biedermann, H. Grote, D. Hathiramani, P. Kornejew,
} D. Rademann, O. Volzke

\author{
Max-Planck Institute for Plasma Physics, 17491 Greifswald, Germany \\ ${ }^{1}$ Forschungszentrum Jülich GmbH, 52425 Jülich, Germany
}

${ }^{*}$ Corresponding author. Tel: +49-3834-88-2534; fax: +49-3834-88-2509.

E-mail address: baldzuhn@ipp.mpg.de

\begin{abstract}
A new plasma fusion experiment, the Wendelstein 7-X (W7-X) has been built in the Max-Planck Institute for Plasma Physics in Greifswald, Germany. A cryostat vacuum tank, shaped like a torus, hosts 70 superconducting magnetic coils to produce a toroidal magnetic field that will confine a fusion relevant plasma. The cryostat tank has a volume of about $420 \mathrm{~m}^{3}$ and is equipped with several hundreds of vacuum ports, feedthroughs and service openings. Nested inside the cryostat is the plasma vessel with a volume of about $80 \mathrm{~m}^{3}$, also shaped like a torus. This plasma vessel is connected to the outside by 254 ports that traverse the cryostat. These ports are needed as access for plasma diagnostics, plasma heating and the supply of cooling media. We report in this paper on the vacuum leak search on the W7-X cryostat, both for the internal piping as well as the integral leak search of the entire vessel against air. This is the initial leak search prior to the first cool-down of W7-X. Emphasis is put on the description of the methods, rather than on the results. The technical commissioning of the cryostat vacuum system within a limited time was the primary task; the emphasis was therefore much more on achieving practical results (the leak-tightness) rather than answering fundamental questions.
\end{abstract}

Keywords: Vacuum leak search; Superconductor cryostat; Magnetic confinement 


\section{Introduction}

Wendelstein 7-X (W7-X) [1,2,3] is a stellarator [4]; a plasma fusion experiment to exploit the potential of controlled nuclear fusion [5] for the production of energy. The plasma is confined by a strong magnetic field inside the plasma vessel which is nested within a toroidal arrangement of magnetic coils. The plasma reaches temperatures up to 100 millions of degrees Celsius. The particular toroidal shape of the magnetic field will minimize the losses of plasma energy and particles, allowing for high performance plasma discharges with a length of up to 30 minutes. W7-X uses superconducting coils for the production of the magnetic field with a flux density of up to $3 \mathrm{~T}$ in the plasma center. For the successful operation of W7-X, a vacuum pressure $p$ in the range $p<10^{-4}$ mbar as insulation vacuum in the cryostat for the superconductor, and $p<10^{-7}$ mbar for the plasma operation in the plasma vessel, are mandatory. Good plasma confinement requires a large experiment; therefore the outer diameter of the W7-X cryostat is about 16 meters.

Fig. 1 shows a sketch of the W7-X cryostat [6] with the coils [7] and the plasma vessel. To have access to the plasma, 254 ports reach across the cryostat vessel, from the outside into the plasma vessel. Those ports are for the technical purpose of heating the plasma with several MW of heating power, the vacuum pumping of the plasma vessel and the water cooling of the plasma facing components, and for the plasma diagnostics. In the future, the use of an in-vessel cryo-pump system is foreseen in the plasma vessel. These pumps have to remove plasma particles (mainly hydrogen and deuterium will be the working gases) during operation. Finally, plasma diagnostics reach through the ports for the purpose of monitoring and analyzing the plasma properties and behavior. These are, for instance, specialized cameras, microwave antennas, plasma probes, gas pressure probes etc. [8]. The torus of W7-X is composed of five almost identical modules. Each of them consists of a $72^{\circ}$ sector. Each module is made of 2 half-modules. The numbering of the half-modules determines the numbering of the cryostat vacuum pumping stations (see below). 


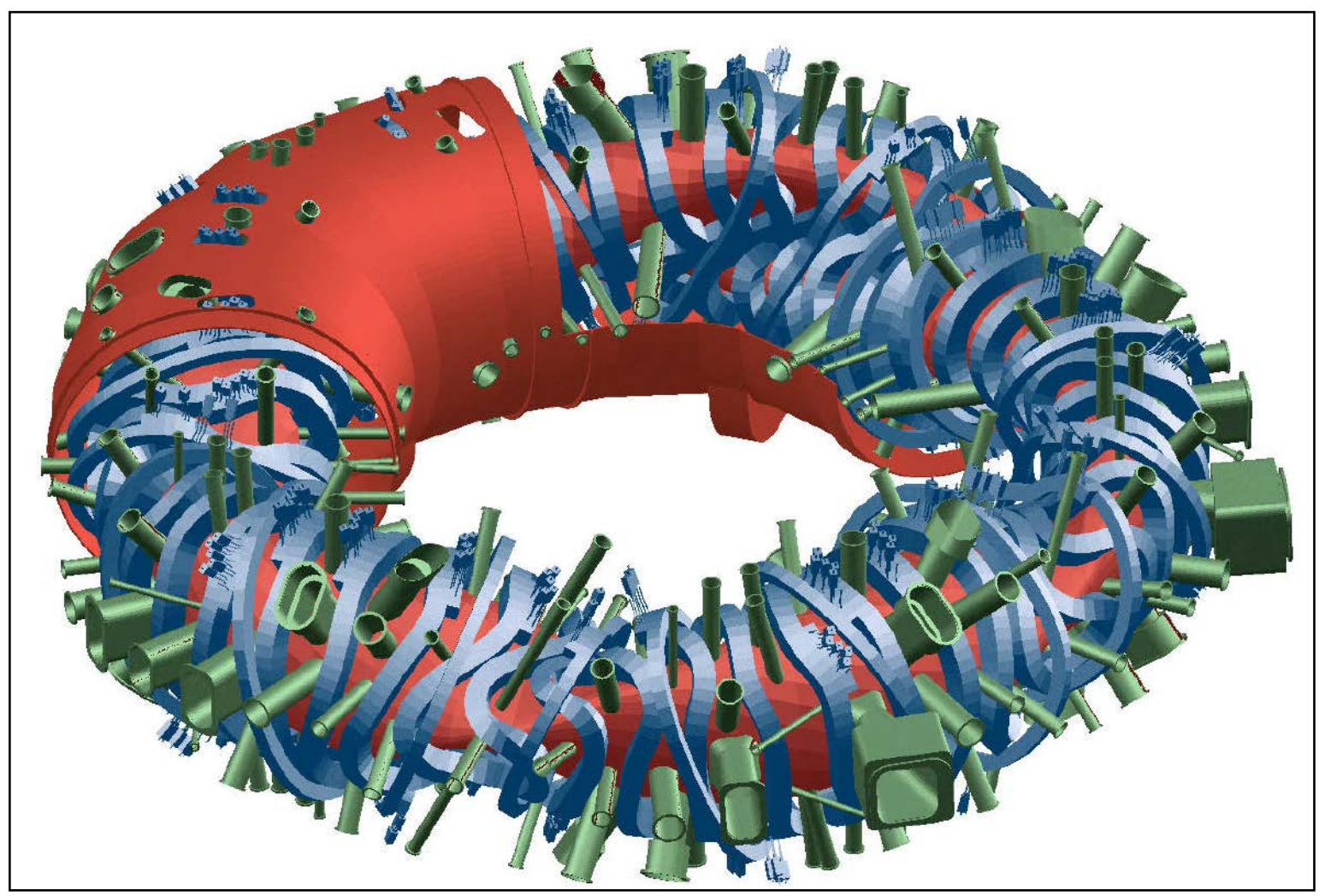

In this first chapter, we give a short description of the vacuum vessels. Seventy

superconducting coils are the most important components in the cryostat. Each of them has an outer diameter of up to about $6 \mathrm{~m}$ and a weight of up to about 5 tons, arranged such that they form the torus. The coils are made of NbTi superconducting winding packages that are cooled by supercritical helium to temperatures between $3 \mathrm{~K}$ and $4 \mathrm{~K}$. Currents of up to $17 \mathrm{kA}$ will be fed to the coils to provide a magnetic flux density of up to $3 \mathrm{~T}$ in the center of the plasma. The coils had been subject to thorough leak tests before [9]. A cryogenic supply line system provides the cryogenic helium, both for the coils and for the radiation shields surrounding the coils. Superconducting current leads, meshed bus bars and specialized joint connections feed the electric currents to the coils. The plasma vessel and the ports to this vessel are covered by water pipes. They will be used for cooling purposes and for the baking of the plasma vessel at $150{ }^{\circ} \mathrm{C}$. A helium cooled stainless steel support ring with a diameter of about $8 \mathrm{~m}$ supports the coils mechanically. That support ring bears a part of the magnetic mechanical forces between the coils during operation, and it has to provide enough flexibility for all mechanical structures in the cryostat. Thus, too large mechanical stresses during the cool-down and due the Lorentz forces will be avoided. Super-insulating multi-layer thin film foil packages improve the thermal insulation of the coils against the inner surface of the vessel being at ambient temperature. Hundreds of thermo-couples, strain gauges, 
mechanical extensiometers, helium flow meters and pressure sensors are distributed in the cryostat to diagnose the system status during operation. A sophisticated quench detection system, utilizing voltage taps along the superconductors, will help initializing a fast current discharge in case of a quench. All sensor cables, supply lines and current leads are fed through ports on the cryostat surface. Fig. 2 shows a sketch of the cryostat surface with its ports, openings, weld seams and access openings.

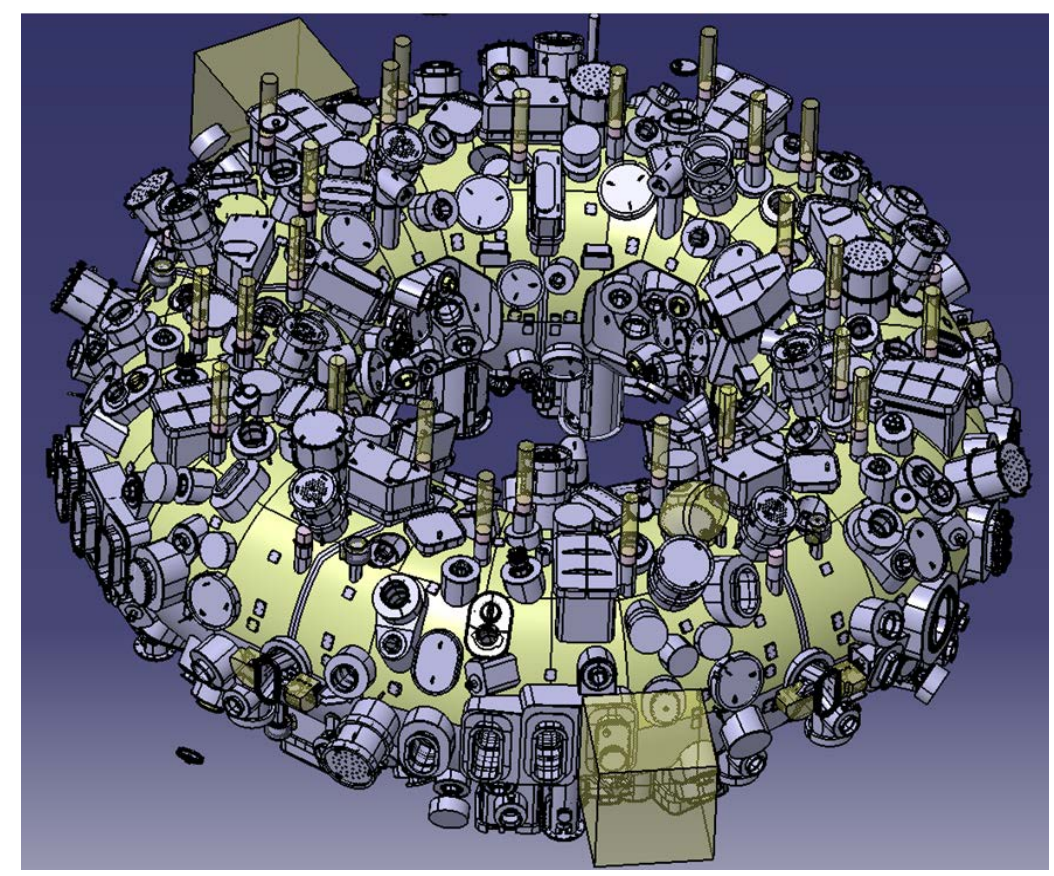

\section{The vacuum pumping systems}

In this chapter we describe briefly the vacuum pumping systems. Five independent vacuum pumping stations pump the cryostat vessel. They are distributed almost evenly around the torus. The distribution is not perfect because of the very tight space situation in the experimental hall. The modules 1-3 have one pumping station each (labeled no. 11, 21, 31), module 4 has none, module 5 has two of them (labeled no. 50 and 51). Each station consists of a Leybold rotary pump D65B in series with a Leybold roots pump WSU251. The roots pump can be by-passed for the operation at high pressure. A Pfeiffer turbo pump HiPace $2300 \mathrm{C}$ is connected in series with the roots and rotary pumps. They are coupled by a tube DN320 with a length of about $3 \mathrm{~m}$ to the cryostat. This large distance is inevitable, because the turbo pumps have to be situated outside the 
magnetic field of W7-X. Only at distances $>3-4 \mathrm{~m}$ from the cryostat, the magnetic field strength is low enough to allow for a safe turbo pump operation. Additionally, the magnetic field configuration of W7-X, required for good plasma confinement, will not be perturbed by components in the pumps with a magnetic permeability larger than one. Gate valves are placed directly in front of all turbo pumps and in front of the cryostat. Fig. 3 shows the pump arrangement for the cryostat. Each of the pumping stations can be operated completely independent from the others. The connection area between the pumping tubes and the cryostat is covered by radiation baffles (chevrons) to minimize the thermal radiation power load to the cold surfaces of the superconducting coils.

In addition to the cryostat pumping system, an interspace vacuum system for bellows and seals is installed. This pumps the intermediate volume between double Helicoflex ${ }^{\circledR}$ gaskets [10] and the gaps between multi-layer bellows. Finally, the plasma vessel will also be evacuated by an independent pumping system using series of rotary pumps, roots pumps and turbo pumps quite similar to those used for the cryostat. 


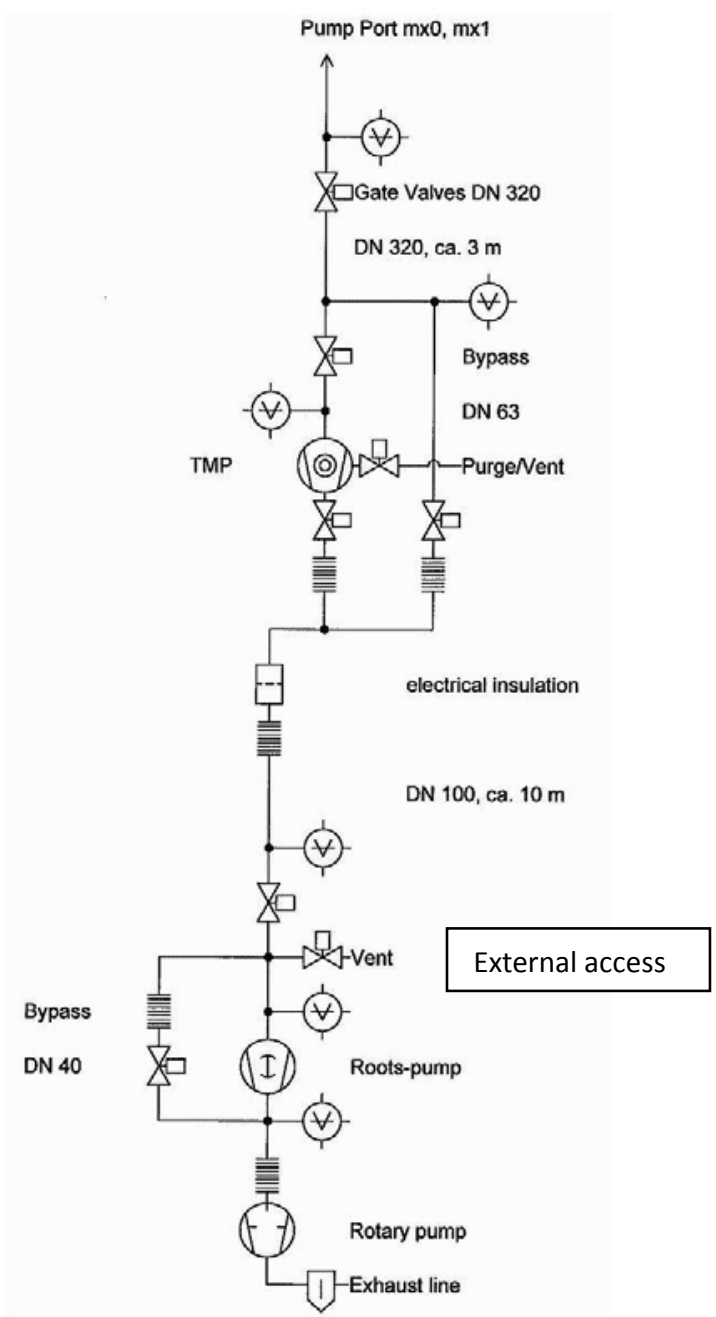

Mass spectra could be measured by a mass spectrometer connected to a differential pumping system with 3 pump stations (each with a rotary pump and a turbo pump). Fig. 4 shows the scheme of the system, which provided a very high flexibility concerning the choice of the pressure at the inlet.

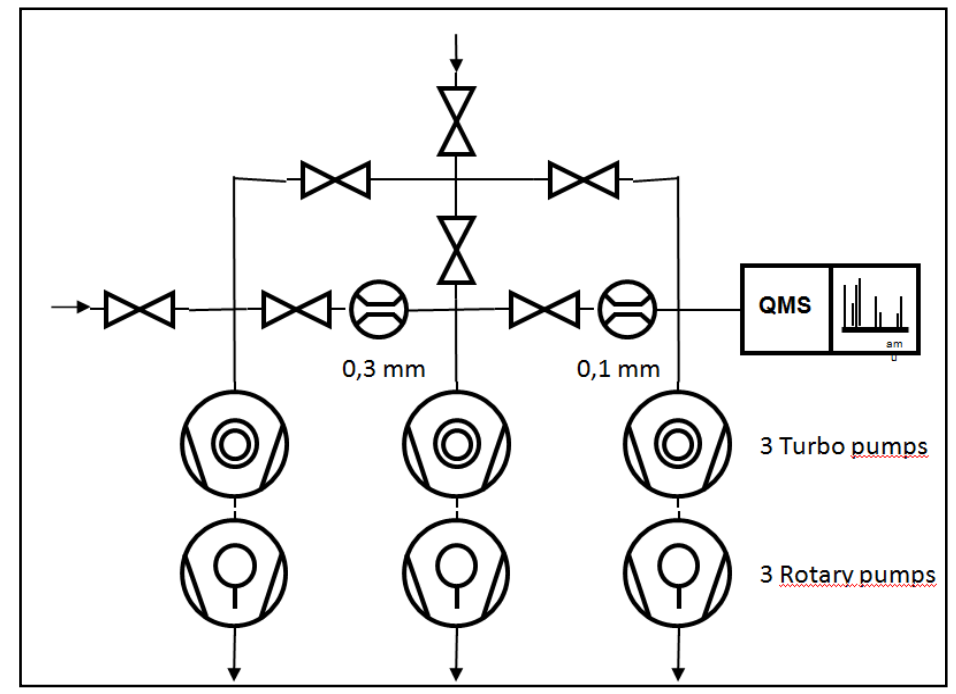


A quadrupole mass spectrometer Pfeiffer Prisma QMG220 was utilized, at higher pressures above $10^{-6}$ mbar operated in the "Faraday mode". At pressures below $10^{-6}$ mbar the "Secondary Electron Multiplier" was utilized to increase the sensitivity.

3. Local leak search procedure during the assembly of the cryostat

Already during the assembly of W7-X, local and individual leak tests were performed on all internal components before and after installation, as far as technically possible. This concerned in particular all pipes for water and helium, with emphasis on weld seams or flange connections. Furthermore, all electrical current leading components, like current joints or the current leads as well as the instrumentation feed-throughs, were subject to a series of leak tests, both under room temperature and cryogenic conditions. This leak search as early as possible seemed mandatory. The later the leak search, the more complicated and time-consuming it appeared. This was due to the complexity of the cryostat, the extremely crowded space situation inside and almost impossible later human access to many components. The criterion of an allowed maximum helium leak rate $<10^{-9} \mathrm{mbar}$ l/s was set as limit for all tests. All coils were tested piecewise before delivery to IPP Greifswald in the cryogenic test facility at CEA Saclay, Paris [11], after the very first acceptance tests by the manufacturer. Besides helium, also $\mathrm{SF}_{6}$ gas was used as leak tracer for these coil tests.

For each tested component an individual leak test chamber had to be built, that fitted exactly to its measures and geometrical shape. Silicone gaskets were cast in individually produced casting moulds. Wacker Elastosil ${ }^{\circledR}$ type M4642 or M4370 was used for the gaskets to guarantee vacuum tightness. This is a 2 compound silicone, that provided leak tight gaskets even a $77 \mathrm{~K}$. The test chambers were manufactured on-demand individually for each sample in the local workshop. Fig. 5 shows a collection of typical test chambers and gaskets [12]. Note that the gaskets are split into two parts, to allow for the installation around components which are already installed. 


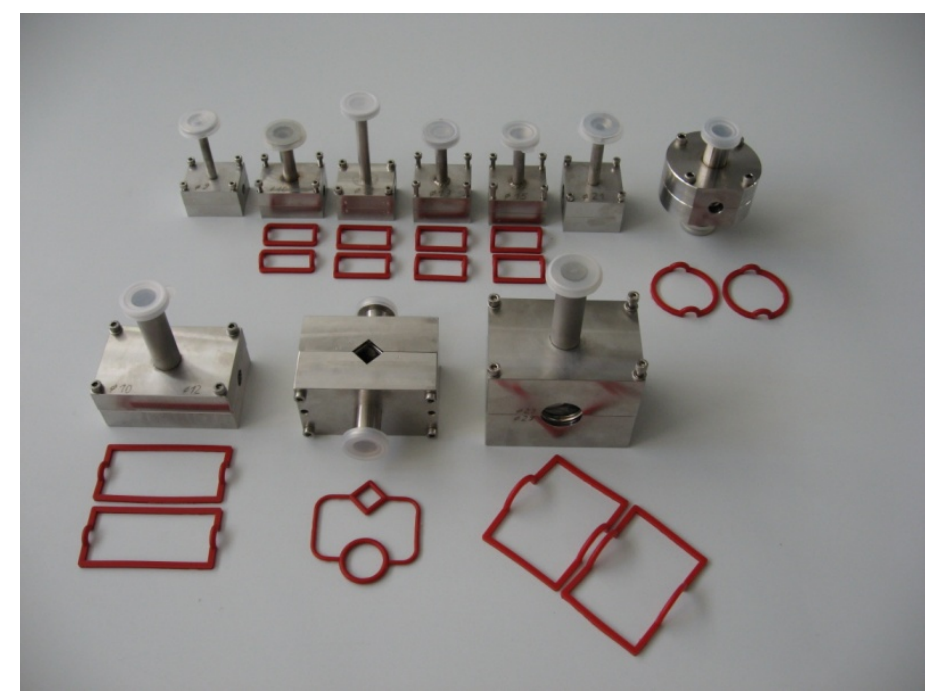

In the simplest case the local test chamber was applied around the sample, evacuated and connected to a helium leak detector. Then the sample was filled with helium at different pressures (up to 90 bar) while the helium flux was recorded. Liquid nitrogen $\mathrm{LN}_{2}$ was utilized in an open bath to cool down the sample for the cold leak tests. One technique employed an arrangement as shown in fig. 6 . The sample was evacuated and connected to the helium leak detector ("Tube for the leak detector"). A temperature sensor was glued or clamped ("T-sensor cable”) directly on the sample. The local test chamber was then situated around the sample. When the testing temperature was reached, the test chamber was evacuated or filled with helium gas alternatively ("He supply pipe”), simultaneously the leak detector reading was recorded. For that type of leak tests, the helium pressure was typically in the range of 1 bar. Vacuum levels of down to $10^{-3}$ mbar could be reached with these chambers.

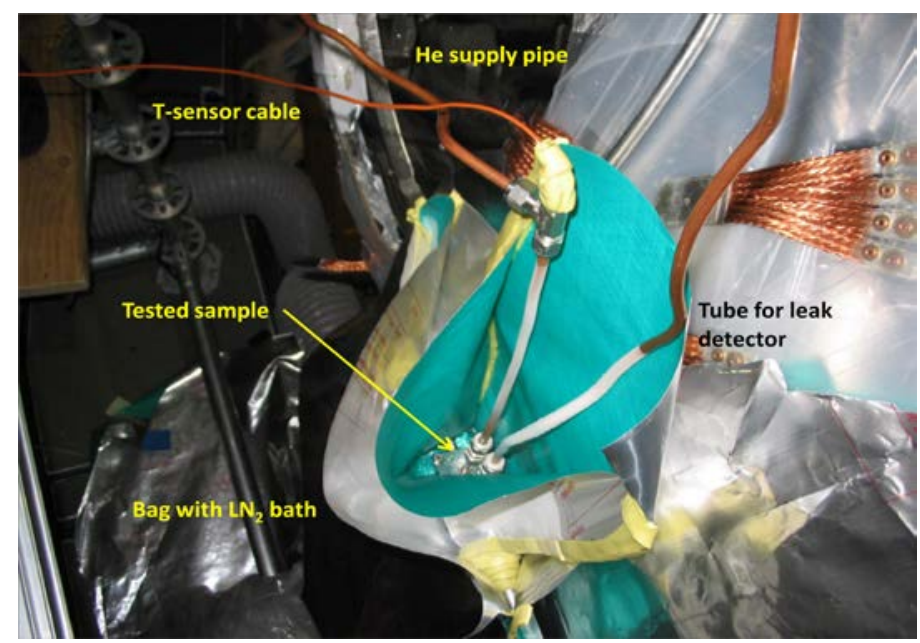

Alternatively, the samples were evacuated and then pre-cooled in a $\mathrm{LN}_{2}$ bath, typically during 10 minutes. After the samples reached the $\mathrm{LN}_{2}$ temperature, they were placed in 
the local test chambers which were connected to the helium leak detector. With still cold sample, helium gas was then applied up to the test pressure, and the leak detector response was recorded.

In some cases the test direction had to be reversed because access was otherwise not possible, i.e. helium had to be applied from outside and the inner volume was evacuated. This is in opposite to the later operation modus, where the helium is inside and the vacuum outside.

\section{Pumping down and leak search on the outer cryostat skin}

In this chapter, the leak search on the outer cryostat skin is described. This comprises all leaks towards the air. The first activity was a measurement of the volume in the cryostat. For that sake, the cryostat vessel was filled with a well defined amount of dry air from bottles. By measuring the bottle pressure before and after the filling, and by recording the pressure in the cryostat, the volume could be determined to about $420 \mathrm{~m}^{3}$. This value could be confirmed by calculations based on the CAD data for the cryostat. To improve the quality of this measurement, it was repeated several times, with a total amount of dry air in the range between 1000 barll and 60000 barl. The pressure in the cryostat was increased very slowly (typically by 1-2 mbar/minute) by throttling the air flow. This minimized the hazard for mechanical structures and bellows resulting from too high mechanical stresses. The gas pressure in the cryostat was measured using a Piza/Pirani transducer (Ilmvac GmbH [13]), the typical pressure increase per measurement was between 3 mbar and 150 mbar.

Fig. 7 shows the time trace of the pressure for the first complete pump-down of the W7$\mathrm{X}$ cryostat. Despite the large volume of the cryostat and the large internal surface with numerous layers of super-insulating foil, the roughing could be accomplished within a few hours by the rotary pumps only. At pressures below 100 mbar the roots pumps were switched on as well, to avoid back-streaming oil vapor into the cryostat and to enhance the pumping speed. During pumping, some rest periods were intercalated to check the sensors and the control electronics, to make mechanical measurements and to do a first estimate of the leak rate and surface out-gassing rate. As no large problems were encountered at this moment, the pumping proceeded with the roots pumps. However, the pressure remained still too large to switch on the turbo pumps during this stage. 


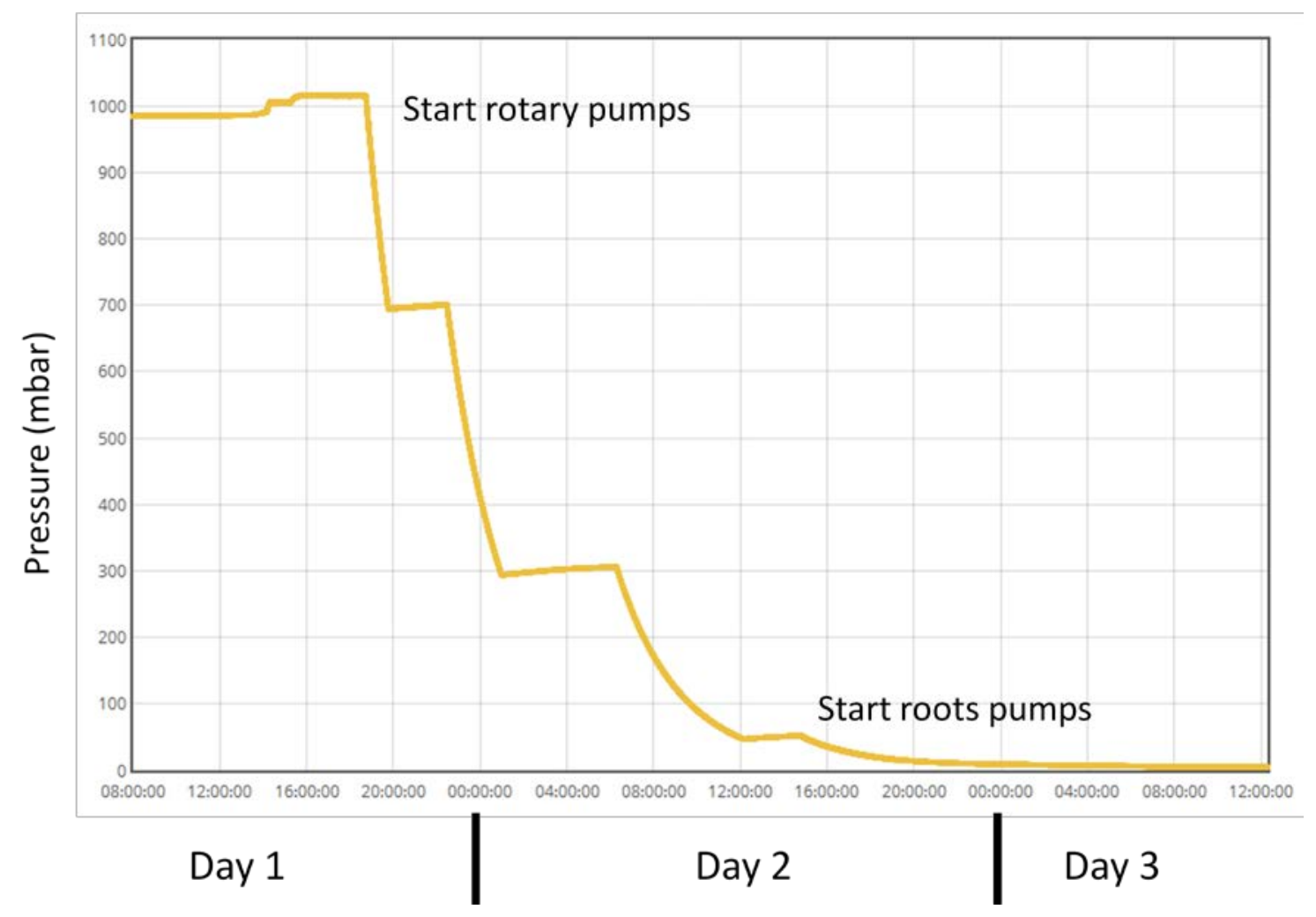

After a few days of pumping with the roots pumps alone, however, the pressure remained above $\approx 0.4$ mbar, indicating the existence of large leaks, and a strong outgassing from the surfaces in the cryostat. This pressure was still too high to switch on the turbo pumps. Taking into account the pumping speed of the roughing pumps, the pressure indicated a total leak rate in the range of $\approx 100 \mathrm{mbar} 1 / \mathrm{s}$. A first inspection and rough leak search by acoustic leak detectors (Ultrasound Solutions, FSR/SDT 270) and additionally helium gas puff with the leak detector revealed large leaks on the so-called five pumping ports (AEP-ports). The reason for these leaks was a couple of seal welds, which were accidentally left open after a technological modification of the port design. This modification was mandatory for the mechanical assembly of the port tubes. A provisional sealing with Tacky-tape $₫[14]$ closed these leaks temporarily and reduced the remaining leak rate considerably, thus confirming their leading role. These leaks were fixed by welding the supporting welds vacuum, making them the new seal welds. The enclosed volume between seal and supports welds after the repair is small compared to the entire cryostat, and the leak rate into the cryostat is large $(\approx 100 \mathrm{mbar}$ l/s). Therefore the hazard of producing a virtual leak is negligible and appears as acceptable. The alternative, namely a further mechanical opening of the gaps in the seal weld by drilling, was rejected because of the too high risk for the cryostat installations behind. 
Learning from that incident, a list of about 170 ports was compiled, which might exhibit similar weld seam problems; therefore these ports were leak searched with priority. It was suspected that problems might arise from damaged seal welds due to the assembly process of the ports (as this happened for the AEP-ports mentioned above), or from the very particular welding geometry. As one potential welding problem, T-seams could be identified. Fig. 8 shows a photograph of such a weld seam. Critical point is the junction, where the two weld seams come together. In fact, seven further major leaks could be detected at such locations by the leak search.

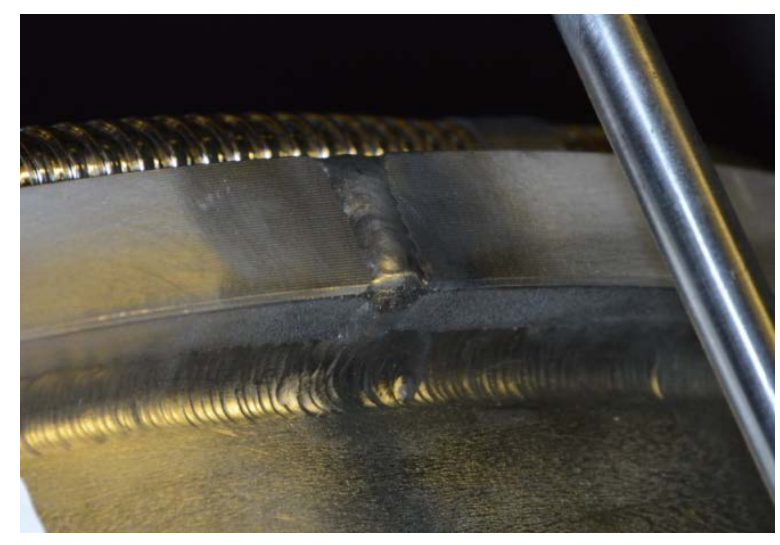

Fig. 9 shows a mass spectrum taken a few days after starting the cryostat pumping, indicating clearly the dominating contribution from leaks to the air. The mass numbers $28 \mathrm{amu} / \mathrm{e}\left(\mathrm{N}_{2}\right)$ and $32 \mathrm{amu} / \mathrm{e}\left(\mathrm{O}_{2}\right)$ are the strongest in the spectrum, the ratio of the ion currents for the mass numbers $18: 28\left(\mathrm{H}_{2} \mathrm{O}: \mathrm{N}_{2}\right)$ is 0.2 . This shows that the air leak rate is still much larger than the out-gassing of water from the internal surfaces.

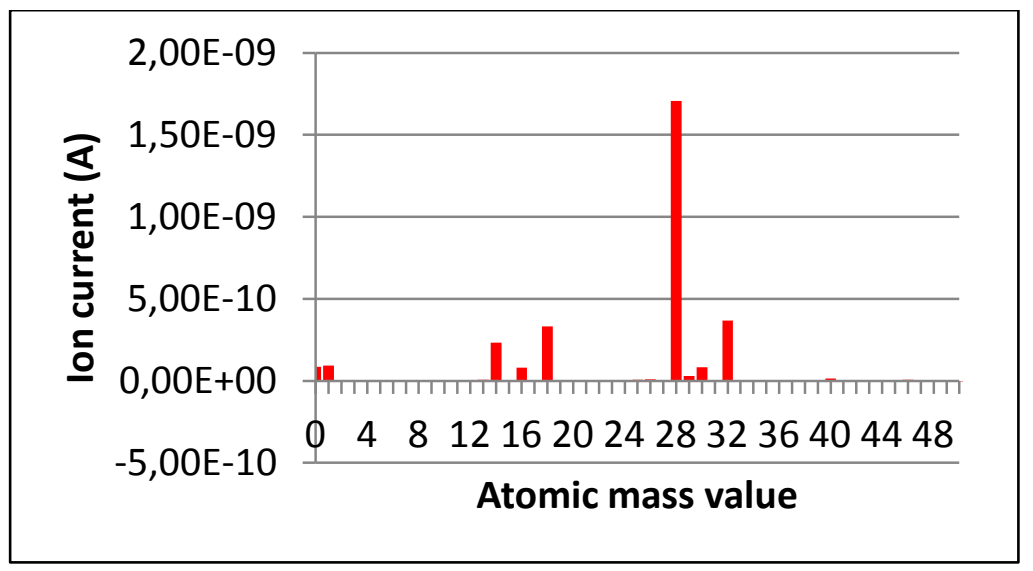

After the leaks in the AEP-ports had been fixed by welding, the remaining leak rate was still in the range of $\approx 30 \mathrm{mbar} 1 / \mathrm{s}$. Because of this rather large leak rate, the leak search was continued by using the acoustic leak detector; however without success. 
After closing the above mentioned leaks, the pressure in the cryostat dropped to the range $\approx 0.1$ mbar, such that the turbo pumps could be put into operation. Consequently, the pressure dropped further to the range $\approx 7 \cdot 10^{-3}$ mbar due to the enhanced pumping speed compared to the roots pumps. The leak search was then continued by helium gas puff to the cryostat skin, with a helium leak detector (Leybold Phoenix L 300) connected to one of the pumping stations. The helium flux into the vessel was recorded electronically as a function of time; a tool that was mandatory for the leak search. Typically, the time delay between the external helium puff and the increase of the helium flux reading ranged between 10 seconds and several minutes. The large size of the cryostat and the long spatial distance between the leaks and the pumping station was responsible for that.

Because of the large number of ports and weld seams, a large quantity of helium was sprayed (typically $10-100$ barl) in a first tour to large areas of the cryostat skin (typically between $4 \mathrm{~m}^{2}$ and $30 \mathrm{~m}^{2}$ ). Thus, large leaks could be determined within that area. If leaks existed, then in further tours the search was refined to smaller areas with smaller helium quantities in order to localize the leak better. Taking into account that the leaks were caused by defects in weld seams with a size of typically a few mm, the search had to be refined in numerous steps with continuously reduced search areas and helium quantities. The local sealing or taping of all weld seams and flange openings with plastic foil was impossible because of their large number; this method appeared as much more time consuming as what we did and was therefore rejected.

During the helium leak search procedure, some unexpected issues had to be tackled. For instance, the helium spraying to large areas of the cryostat surface rather frequently overlooked leaks. Obviously air flows in the experimental hall blew away the helium that was puffed to the cryostat. The air flows were produced by local heat sources (power supplies etc.), fans or the air conditioning. Therefore, the leak search had to be repeated frequently in some areas, once with the assumed disturbing sources on, and once off. In some cases "ghost leaks” were detected. Those are leaks, were the leak detector recorded a clear increase of the helium flux after puffing helium to a certain port or weld seam. These "ghost leaks” could be reproduced very well several times, even their size could be measured with good reproducibility. However, after repetition of the leak search some days later at the same port or weld seam, the leaks seemed to have disappeared. In particular, when it was tried to fix the leaks by welding, no success 
was visible. Regularly, a real leak could be found in vicinity to the apparent "ghost leak" when the leak search continued. Normally, they were located upstream the "ghost leaks”. The largest distance ever observed between a "ghost leak" and the real one was about 5 meters. Obviously, air currents or the helium convection in air carried away the helium cloud from the search location to the real leak. Only very thorough and strict documentation during the leak search helped us to discriminate between "ghosts" and real leaks. The very simple means of drawing maps of the cryostat surface with all findings during the search turned out as essential for that purpose.

Fig. 10 shows an example of the helium flux time traces when a large leak $(\approx 10$ mbar l/s) was detected. For that example, the time delay between helium puff and detector response was in the range of $\approx 30-90$ seconds.

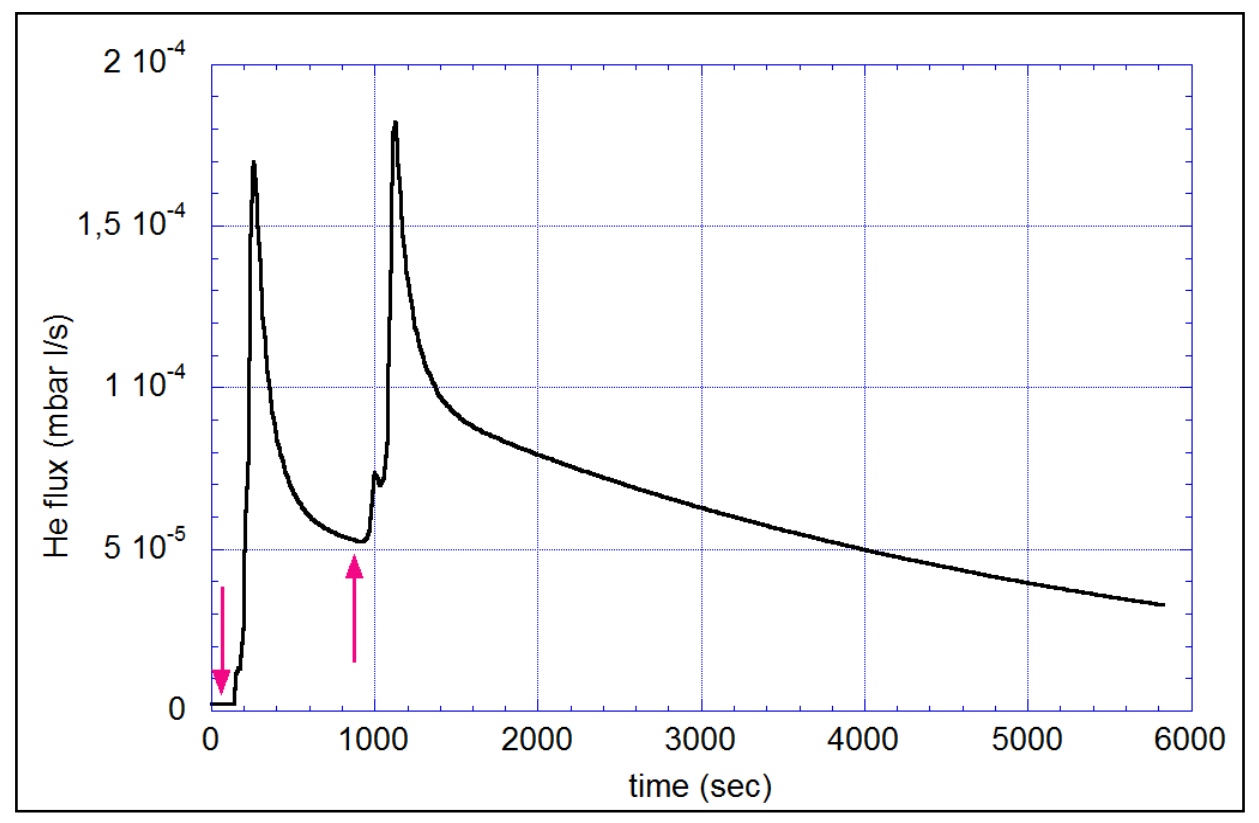

After localizing and welding the leaks one by one, the pressure in the cryostat could be reduced more and more, until after about three months of leak search the pressure was down to $2 \cdot 10^{-4}$ mbar with all 5 pumping stations in operation. The major obstacle to obtain lower pressures was, as expected, the water adsorbed to the surfaces in the cryostat.

The leak detector was always connected to one pumping station, therefore only an unknown fraction of the total helium flux was measured. In order to measure the total air leak rate of the cryostat, and to evaluate the size of each newly detected leak, it was mandatory to perform an absolute calibration of the helium leak detector. For that purpose a well-known calibrated leak was connected to another pumping station, and 
the ratio between calibrated leak rate and measured leak rate was determined. This ratio was strongly pressure dependant. For cryostat pressures in the range of 0.1 mbar it was in the range of 370 and dropped down to about 50 for pressures in the range $2 \cdot 10^{-4}$ mbar. Obviously, the effective pumping speeds of the pumping stations and the leak detector pump are functions of the pressure. This results in a variation of the partial gas flux to the leak detector, in particular close to the transition between a more viscous and a more molecular flow. To cover the wide range of cryostat pressures and leak sizes during the leak search, several calibrated leaks were utilized in the range between 1 mbar $1 / \mathrm{s}$ and $10^{-4} \mathrm{mbar} \mathrm{l} / \mathrm{s}$. When a new leak was detected, then the individual leak size was measured by gluing a little plastic bag around the leak position, filling it with helium and waiting for a stabilization of the leak detector signal at the new level. Finally, the measured ratio from above was multiplied to the leak detector reading to obtain the calibrated value.

Fig. 11 shows a mass spectrum, taken after all major leaks had been sealed. Taking into account the effective pumping speed of the five turbo pumps of about $\approx 500 \mathrm{l} / \mathrm{s}$, this means that the net gas flux (remaining leaks plus out-gassing) was $<0.2 \mathrm{mbar} \mathrm{l} / \mathrm{s}$ at that stage.

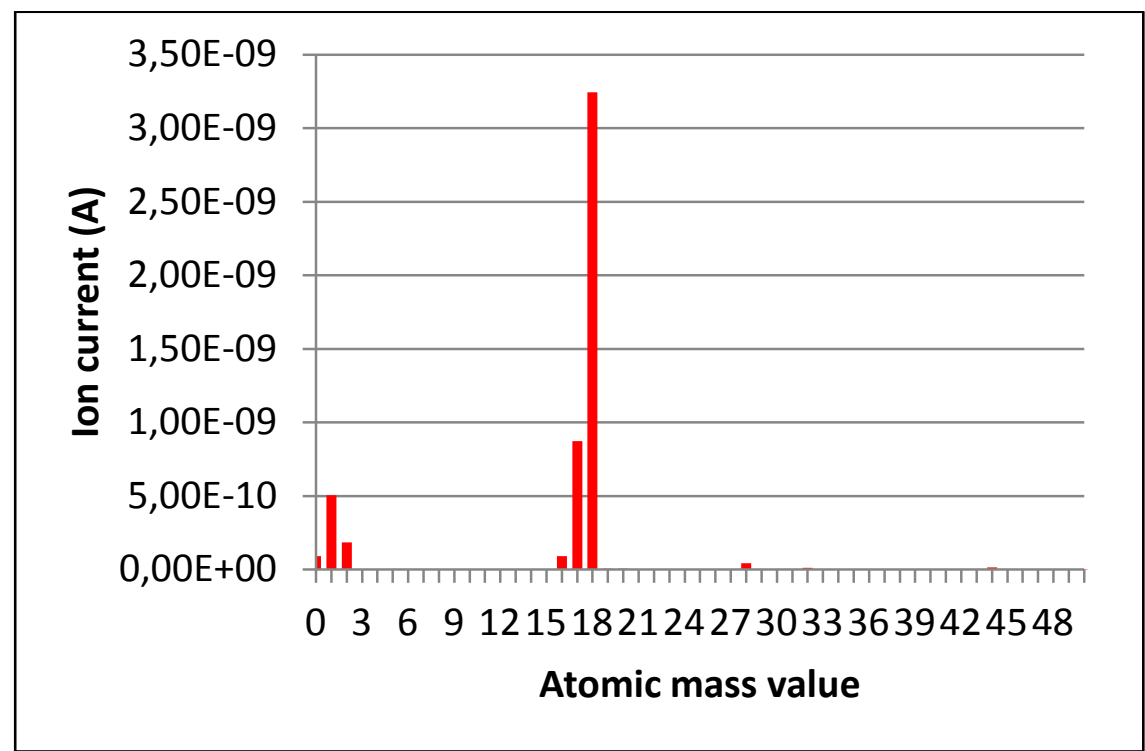

After a few weeks of pumping, the mass spectra indicated that a factor of more than 400 of this gas flux came from the water out-gassing. Hence, the effective remaining leak rate to the air was $<5 \cdot 10^{-4}$ mbar $1 / \mathrm{s}$. This rate stems from leaks to air which had not yet been detected and fixed. 
The above measured leak sizes were confirmed by the following method: in a first phase I, the cryostat was evacuated with the turbo pumps. The ion current ratio 18:28 $\left(\mathrm{H}_{2} \mathrm{O}: \mathrm{N}_{2}\right)$ was determined during this phase by the mass spectrometer, providing in this particular case a value of 200. The cryostat pressure was measured using a Penningsensor as a function of time. During phase II, all five gate valves to the pumps were closed and a calibrated leak was opened, leaving nitrogen into the cryostat. The nitrogen leak rate was measured independently before in the laboratory to $3.2 \cdot 10^{-2} \mathrm{mbar} \mathrm{l} / \mathrm{s}$ for $\mathrm{N}_{2}$. The gas sources were now composed of the (known) calibrated leak, the (unknown) air leak plus the out-gassing water. During a third phase III, the valve to the calibrated leak was closed, leaving only the unknown air leak rate plus the water out-gassing. During the three phases, the mass spectra time traces were recorded, in particular for 28 $\left(\mathrm{N}_{2}\right)$ and $18\left(\mathrm{H}_{2} \mathrm{O}\right)$. The vacuum pressure in the cryostat was recorded as well. Fig. 12 shows the time traces of the mass spectrometer ion currents for water and nitrogen.

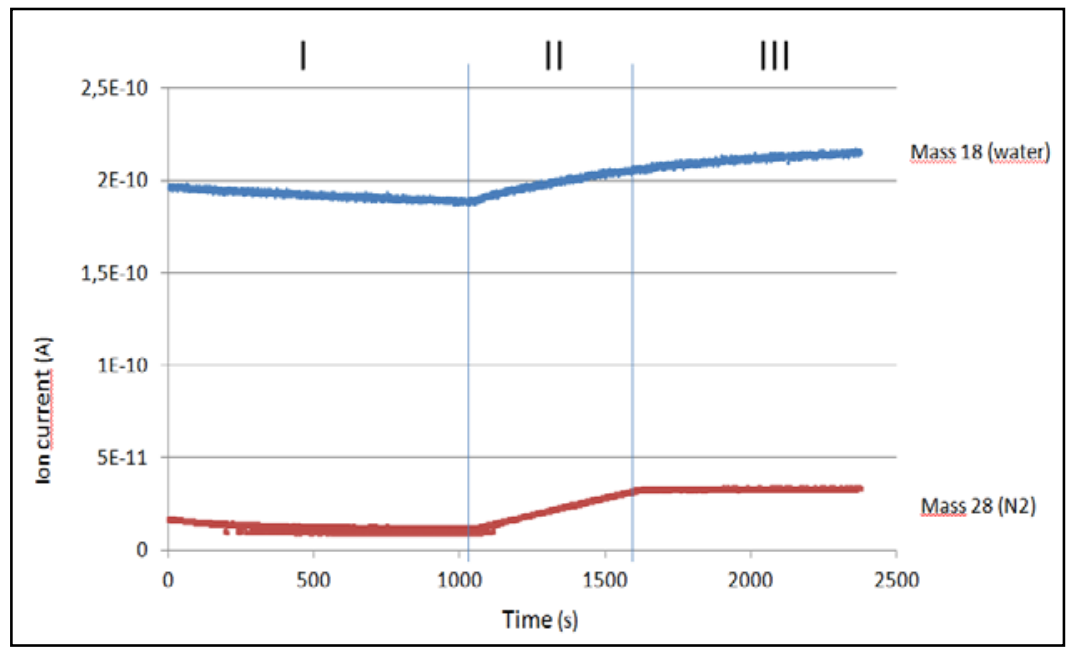

Linear fits to the time traces were done to evaluate the temporal increase of the ion currents for the three phases I-III and the pressure in the cryostat. The numerical comparison provided for the size of the leaks to the air results to $4.6 \cdot 10^{-4} \mathrm{mbar} \mathrm{l} / \mathrm{s}$. The comparison to the calibrated leak rate, by comparing the temporal current increases during phases II and II provided $9.6 \cdot 10^{-4} \mathrm{mbar}$ l/s for the air leaks. This value is roughly by a factor of 2 larger than the first one. That deviation could be explained by measurement errors using the Penning-sensor. In addition the time traces show slight deviations from a strictly linear behavior, a result of the fact that obviously the outgassing rate is a function of the pressure. Finally, the mass spectrometer calibration 
factors between the measured ion currents and the partial pressures, compared for the masses 18 and 28, might also show a dependency from the pressure. However, the exact numerical size of this dependency is unknown.

During the last weeks of pumping, the water partial pressure became more and more dominant. To remove the water from the surfaces efficiently, purging of the cryostat with a dry gas (air) was tested. To minimize the stress excursions for the mechanical components, in particular for the bellows, only one test with 50 mbar was performed. The cryostat was kept for 24 hours under 50 mbar pressure. However, after pumping down again, the measurements of the cryostat pressure and the mass spectra showed that the water partial pressure had not been reduced, compared to the situation before. Therefore, the idea of more purging cycles was discarded. It is assumed that the applied air pressure was too small to show a drying effect.

In order to monitor roughly the amount of water, which is pumped out of the cryostat by the vacuum pumps, little water tanks are installed in the vacuum pump exhaust gas pipes. In these tanks, water and oil can condense after the exhaust gas left the rotary pumps. On a weekly basis these tanks are drained and the quantity of collected water is measured. Typically between 20 and $60 \mathrm{ml} /$ day water can be found, so far. During the last two months of pumping time, no clear decreasing trend could be observed. One has also to take into account, that an unknown quantity of water vapor is carried away with the exhaust gas.

As expected, it needed several iterations of leak search to find all relevant leaks. The combination of the helium leak detector and the mass spectrometer allowed for conclusive indications, where leaks might be located. In the first iterations, large leaks were found, the smaller ones remained undetectable until the repair of the large leaks was done. This iterative process was very time consuming because of the long response time of the leak detector, and long pumping times after the cryostat was polluted with helium. The "ghost leaks" were hard to identify, because they depended on external boundary conditions like the air conditioning of the experimental hall, or the location of fans for large power supplies, that were beyond the direct control of the leak search group. Unfortunately, the leak search was restricted to access ports, which are connected to the five pumping stations. Independent valves on access ports, for instance to connect a calibrated helium leak directly to the cryostat volume, are recommended and will be installed later. 


\section{Leak search on the water pipes}

For the purpose of water cooling and baking of the plasma vessel at $150{ }^{\circ} \mathrm{C}$, a large amount of water pipes are running through the cryostat. The estimated total length of all pipes is in the range of $10 \mathrm{~km}$. About 3000 pipe weldings connect all components with each other. Those pipes with their weld seams had to be leak tested as well. To do so, it was decided to use neon gas because several other leak search activities with helium were done around the cryostat at the same time. But the leak search groups had to discriminate between the activities on the water pipes and all the other installations. Fortunately, the water piping manifold could be divided by valves into about 50 individual circuits, such that each section could be leak tested separately.

Before the leak search, only air or nitrogen had been filled into the pipes. For the tests, each circuit was filled individually with a partial pressure of about 2 bar neon, one after the other. The mass spectrometer was connected to the pumping station no. 50 . The ion currents for the two neon isotopes $\left({ }^{20} \mathrm{Ne}\right.$ : $90.5 \%$ natural abundance and ${ }^{22} \mathrm{Ne}: 9.25 \%$ natural abundance) were recorded as a function of time. Fig. 13 shows one example of the time traces during one leak search period. Each vertical bar shows the dedicated moment of filling one circuit with neon. Some constant neon background could be seen, as well, presumably from the air leaks which had not yet been found at the moment of these measurements, or from out-gassing of the surfaces. Obviously, no additional neon could be detected, leaking out of the water pipes. It was expected to observe a time dependent response of the ion currents for neon in case of a leak, but all water pipes were leak tight. Recording simultaneously the time trace of ${ }^{40} \mathrm{Ar}(\mathrm{m}=40 \mathrm{amu} / \mathrm{e})$ was essential to assure that no traces of ${ }^{40} \mathrm{Ar}^{++}$added to the neon signal ${ }^{20} \mathrm{Ne}$ at mass number 20. It can nevertheless be suspected that some fraction of the constant neon signal at $\mathrm{m}=20 \mathrm{amu} / \mathrm{e}$ was produced by ${ }^{40} \mathrm{Ar}^{++}$, however this did not interfere with the leak search. For control purposes, several other ion current traces were recorded as well. 


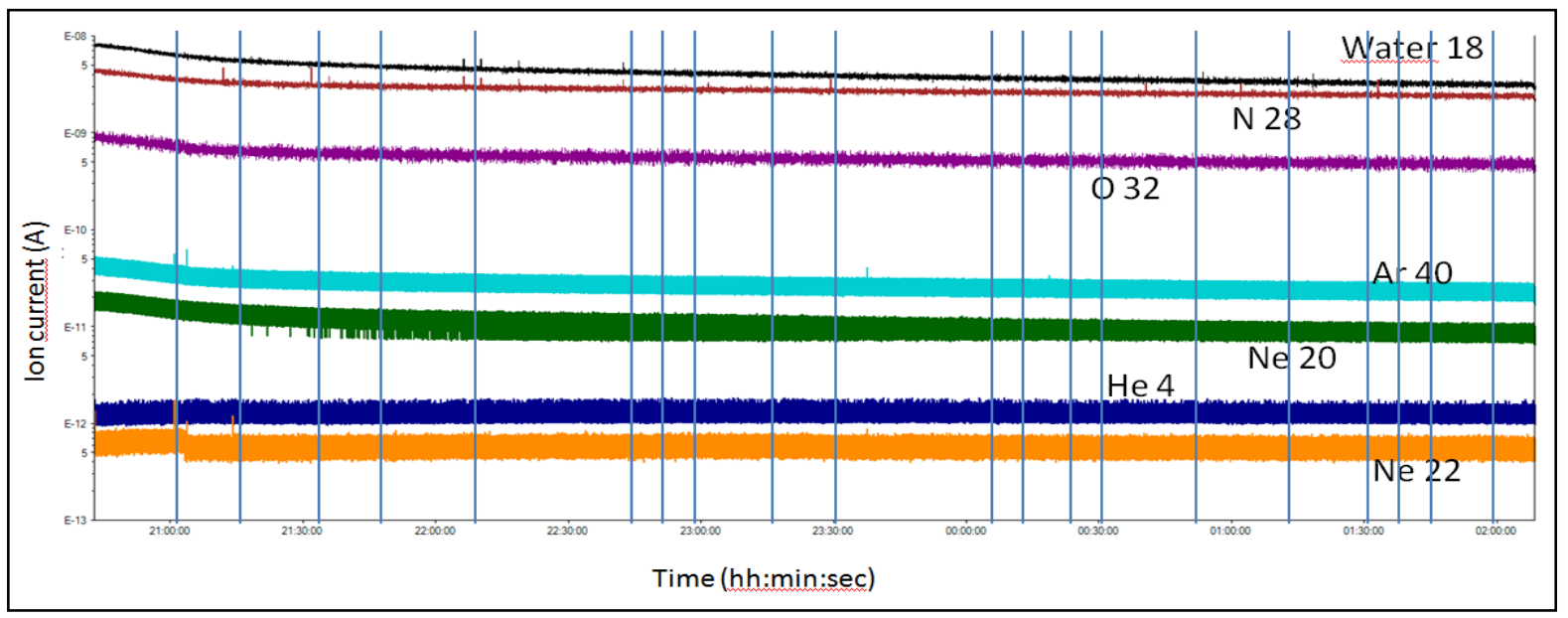

The finding, that all water pipes were leak tight, could be confirmed some weeks later, when all of them were filled with water at a pressure of 12 bar. Each time, when one of the pipes was filled for the first time with water, the mass spectrometer time traces were observed, in particular for the mass numbers 16-18 amu/e. No traces of additional water could be found in the cryostat vacuum. The exchange of helium by neon for the leak detection was a helpful technique to discriminate between different piping systems during parallel activities. During the helium leak search on the cryostat skin, the response of the helium leak detector and the mass spectrometer were compared, indicating a slightly smaller sensitivity of the mass spectrometer against the tracer gas. It is estimated that the mass spectrometer sensitivity for neon might be roughly by a factor of 10 smaller than the leak detector's sensitivity for helium. Therefore the leak search using neon might be suitable only for larger leaks, in the case of doubts the leak search has to be refined using helium.

\section{Leak search in the plasma vessel}

A large surface of the cryostat is formed by the plasma vacuum vessel, sitting nested inside the cryostat. This surface with all its ports was subject to the leak search, too. Because of the continuous assembly activities inside the plasma vessel, human access was possible. The acoustic leak detector and the helium leak search method were utilized again, just as for the outer cryostat skin. However, the acoustic leak detector did not help very much in detecting new leaks in this case; only large leaks ( $>0.1 \mathrm{mbar} \mathrm{l} / \mathrm{s}$ ) that were found by helium leak search before, could be confirmed by the acoustic leak detector. This was nevertheless a helpful method to quickly discriminate between 
"ghost leaks" (see above) and real ones, in particular in the closed volume of the plasma vessel, where puffed helium remained confined (see the remarks below). A real handicap was the fact that the plasma vacuum vessel forms an enclosed volume which is encapsulated by the cryostat vessel. In addition: to protect it against dust and dirt from outside, all ports were closed by air tight plastic caps or the port flanges during the assembly of W7-X. Therefore any puffed helium remained for hours inside the vessel and increased gradually the helium background. Only thorough venting could minimize this effect.

Fig. 14 shows one fifth of the W7-X plasma vessel torus. Inside, roughly the same technique was employed for the helium leak search as for the cryostat outer skin. In a first turn, a larger amount of helium (1-5 mbar l/s) was puffed to a larger area (2-4 $\left.\mathrm{m}^{2}\right)$ to observe whether there are leaks. If yes, after some recovery time the search was refined with smaller quantities of helium on smaller areas, provided the helium background was then small or stable enough.

All leaks that could be detected were situated in the weld seams on the bellows of the ports. The largest one, with a leak rate of $0.1 \mathrm{mbar}$ l/s, was situated just on the welding seam between the bellows and the port tube wall. Those bellows are mandatory to allow for a certain mechanical flexibility of the entire machine when the individual vacuum chambers are evacuated (plasma vessel and cryostat, respectively), the superconducting coils are cooled down, the plasma vessel will be baked, or when the electric current is applied to the coils.

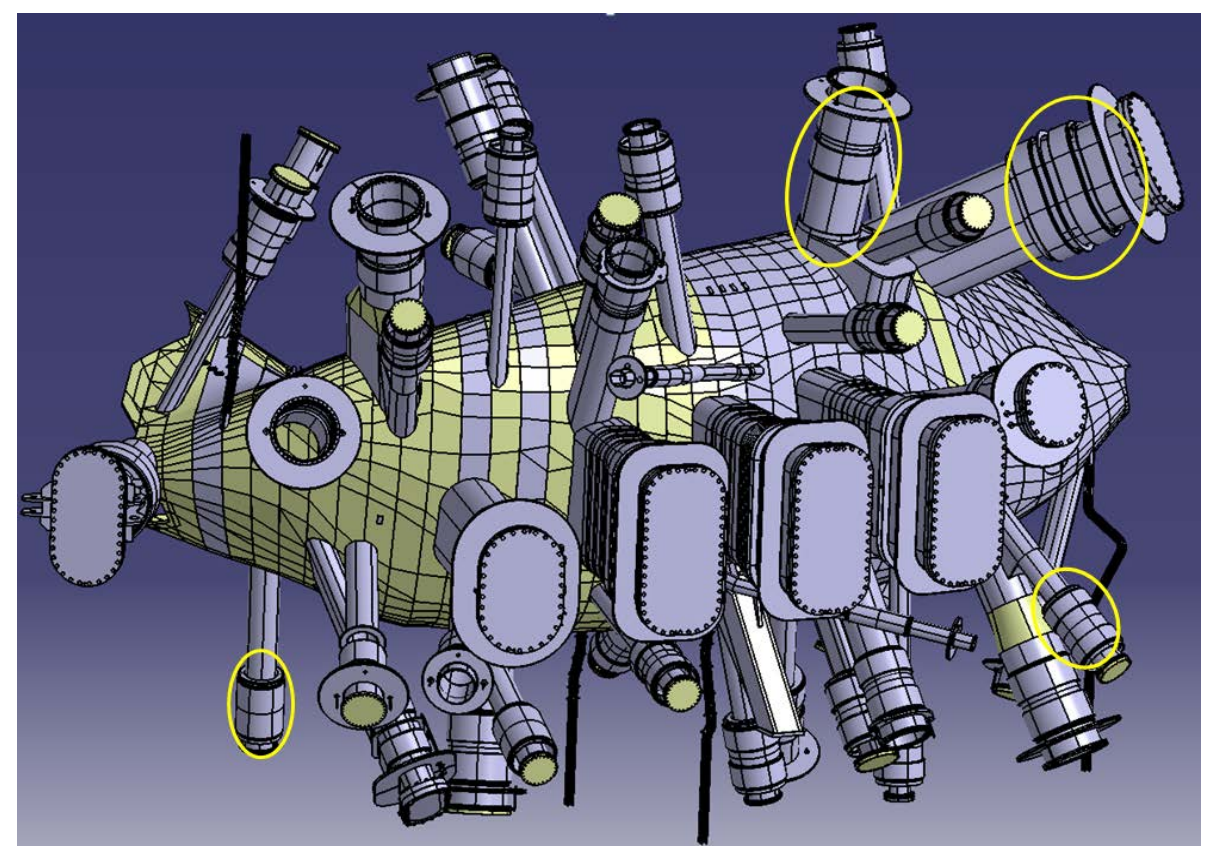


All leaks found in the plasma vessel were fixed, although they were considered as not that critical for the W7-X operation: if both, the plasma vessel and the cryostat vessel, are evacuated, leaks between them will play no dominating role any more.

7. Leak search in the cryogenic pipes

The cryogenic helium is provided by a wide system of cryo-pipes to the superconductors, the current joints, the current bus-bars and all cooled mechanical structures. Again, the total length of all pipes is in the range of $10 \mathrm{~km}$. For instance, the 70 superconducting coils are all connected together in one mesh, without separation valves between them. In total, 5 individual helium pipe meshes exist, partly connected, partly they can be separated by valves. Fig. 15 shows, as one example, a part of the coil helium supply mesh. It was clear from the beginning on, that the localization of any possible leaks will remain very rough, because only 5 pumping stations exist around the cryostat. Therefore, each station has to pump a large volume with numerous cryogenic installations inside. The pumping speed for the five pumping stations is, compared to each other, (within the measurement errors) the same. This was confirmed by pumping the cryostat only with a single station for 1-2 days, respectively, until the pressure saturated. The obtained cryostat pressure was for all 5 pumping stations in the same range.

The leak search under room temperature is essential for the cryostat performance, because each leak could deteriorate under cold conditions drastically, and the cold helium under supercritical conditions will expand by roughly a factor of 230 when penetrating a leak into the cryostat vacuum. The W7-X superconductors will be operated at a helium pressure $>4$ bar; thus always above the critical pressure. 


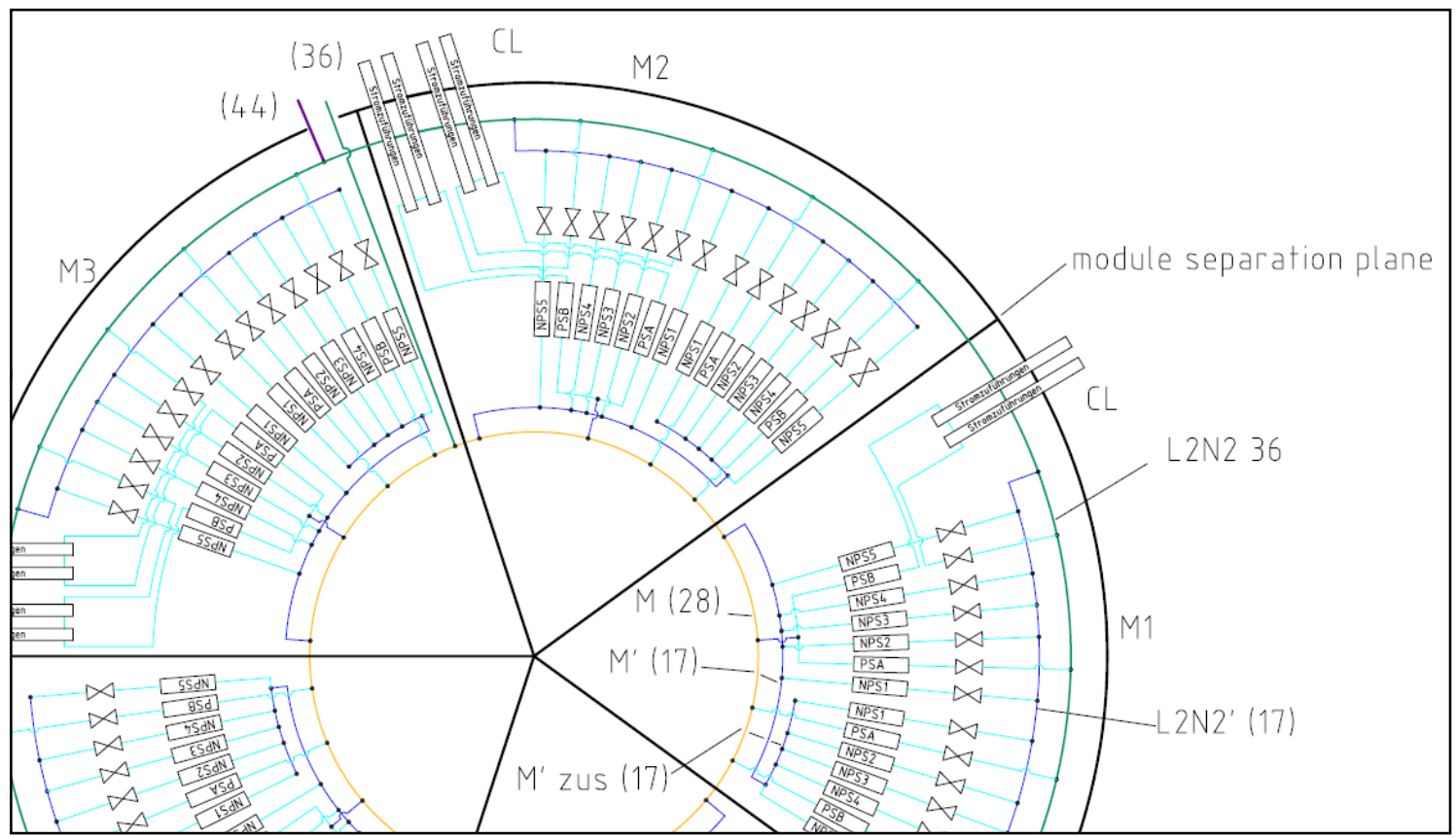

When helium at room temperature was applied at a pressure of 4 bar to the cryo-pipes, an increase of the helium flux could be observed on the leak detector. The direct comparison to a calibrated leak, connected to the cryostat, indicated an integral room temperature leak rate of $3 \cdot 10^{-5} \mathrm{mbar}$ l/s. However, for the later repair the exact location of the leaks has to be known, and therefore several localization methods were tried. Those are described now. In a first step, the helium pressure was increased in each mesh individually and the leak detector response was observed. Fortunately, only one out of the 5 meshes showed a leak, the others were tight. The leaking mesh is labeled "L1 L2N2 - K” and consists internally of three ring lines labeled “L1”, L2N2” and "K”, each of them with an individual inlet valve. The ring lines are connected internally to each other by cooled components of higher hydraulic resistivity, for instance by the coil winding packs. Unfortunately, no parts can be separated within this mesh by valves. Despite of that, it should be determined which of the three ring lines is leaking. Therefore, in a second step, the helium pressure at the inlet of this mesh was increased instantaneously from 4 bar to 6 bar, and then the input valve was kept 30 seconds open. Again, the leak detector response was recorded and the time traces compared afterward. The temporal pressure increase was applied separately to each of the three ring lines, the other two inputs remained closed, respectively. The fastest response could be seen on the ring line "L2N2". Therefore it is assumed that the leak(s) are situated there. Fig. 16 shows the time traces of that measurement. 


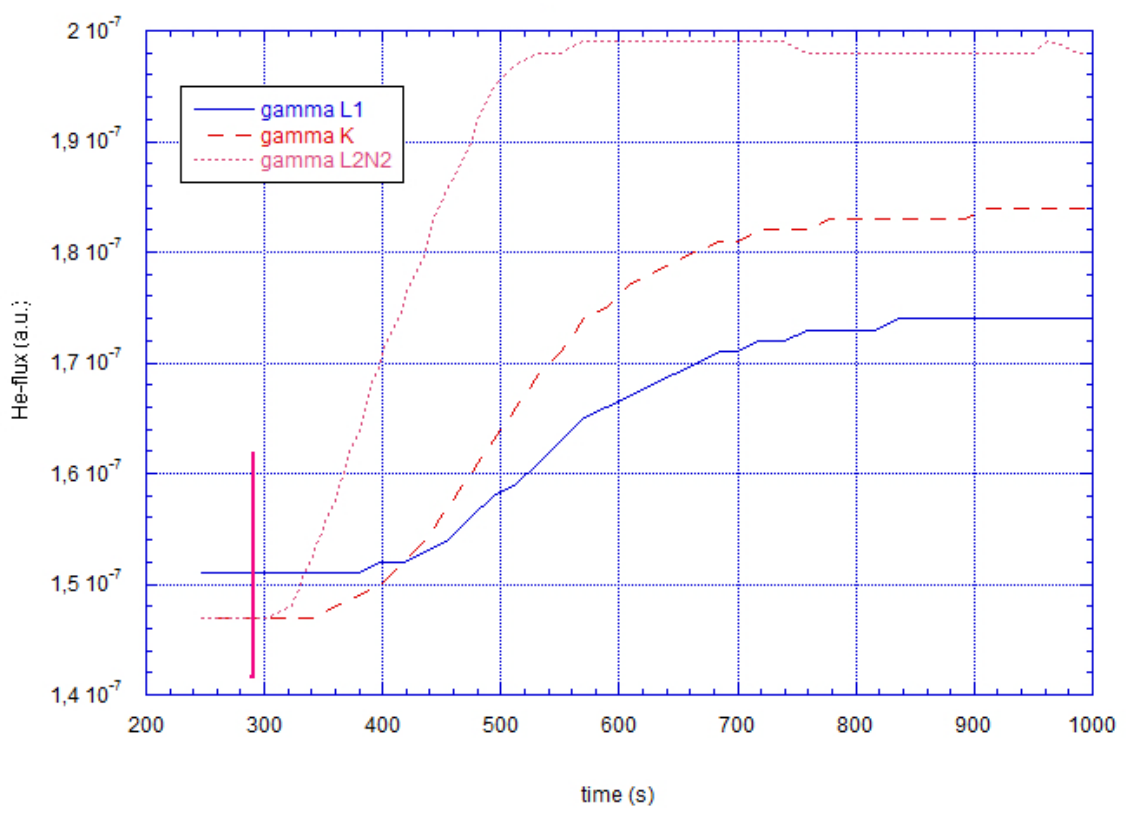

Due to different hydraulic resistances for the inlet of the three ring lines, the final pressure in the entire mesh was different for the three trials shown in fig. 16. Therefore, the saturation helium flux is also different for these three cases. As the measurement of the leak rate was irrelevant for the localization procedure, this circumstance did not disturb.

After localization of the leak to the ring line "L2N2", it should now be identified in which module the leak(s) might be concentrated (or a single leak might be located). Therefore 5 leak detectors were connected to the 5 pumping stations, and a helium pressure step was applied to line “L2N2”. The leak detector time responses were recorded. Fig. 17 shows some results for two test runs with slightly different helium input pressures. The delay times between the start of the helium pressure step and the increase of the leak detector are shortest for the pumping stations no. 21 and 50, possibly indicating that these pumping stations are closest to the leaks. That result of this first method required another confirmation to enhance the reliability, therefore two further methods were tried. 


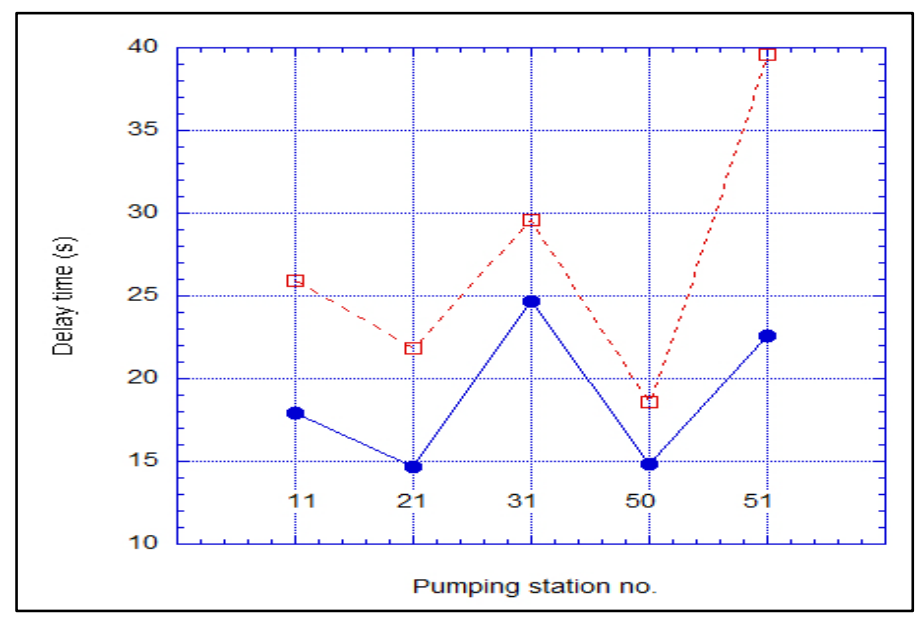

For the second method, only one leak detector was connected to one of the five pumping stations. All five pumping stations were connected to the cryostat. The helium flux was recorded for constant pressure in the cryo-pipes, stationary conditions provided. Then one of the remaining four pumping stations was disconnected from the cryostat by closing the corresponding gate valve, until again a stationary helium flux signal was observed. Due to the reduced integral pumping capability, the helium signal increased. Then this valve was opened again and the next one was shuttered off. That way 4 helium flux maxima were recorded. The idea was that the pumping station next to the leak(s) might see the highest influx of helium from the leak and pump away more efficiently than the other pumping stations. Closing the station in vicinity to the leak might thus lead to the highest increase of the detectable helium for the leak detector. Unfortunately, the outcome was not as clear as desired. Fig. 18 shows one example of a time trace of the helium leak detector signal, when the leak detector was connected to station no. 11.

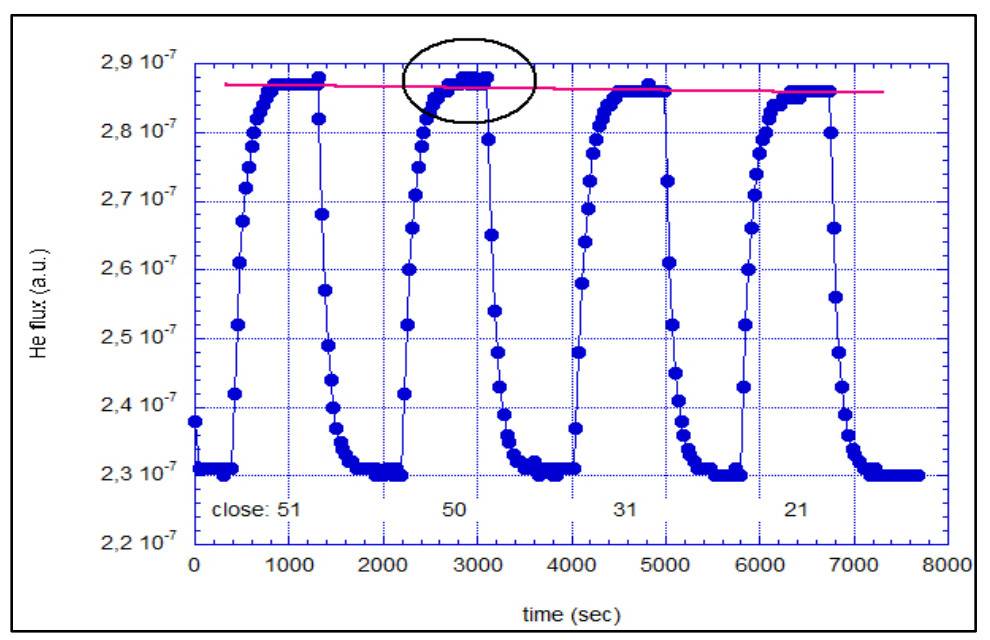


The table I shows all results collected. A + sign indicates a higher helium flux for the corresponding station closed, 0 means that the helium flux is comparable to the others, a - sign indicates a lower helium flux. Albeit very noisy, only the station no. 50 shows increased helium fluxes, possibly indicating that a leak might be in vicinity. This corresponds to the result from the delay time measurement. However, the position no. 21 remains here unremarkable, presumably because the noise level is rather high and/or the signal level is very low compared to it.

\begin{tabular}{|l|l|l|l|l|l|l|}
\hline $\begin{array}{l}\text { Leak } \\
\text { detector } \\
\text { connected to } \\
\text { station no: }\end{array}$ & $\begin{array}{l}\text { Station } 11 \\
\text { closed }\end{array}$ & $\begin{array}{l}\text { Station 21 } \\
\text { closed }\end{array}$ & $\begin{array}{l}\text { Station } 31 \\
\text { closed }\end{array}$ & $\begin{array}{l}\text { Station } 50 \\
\text { closed }\end{array}$ & $\begin{array}{l}\text { Station 51 } \\
\text { closed }\end{array}$ \\
\hline 11 & & $\mathbf{0}$ & $\mathbf{0}$ & + & $\mathbf{0}$ \\
\hline 21 & $\mathbf{0}$ & & $\mathbf{0}$ & + & $\mathbf{0}$ \\
\hline 31 & $\mathbf{0}$ & $\mathbf{0}$ & & $\mathbf{0}$ & $\mathbf{0}$ \\
\hline 50 & $\mathbf{0}$ & - & $\mathbf{0}$ & & $\mathbf{0}$ \\
\hline 51 & $\mathbf{0}$ & $\mathbf{0}$ & $\mathbf{0}$ & + & \\
\hline
\end{tabular}

The third method is complementary to the second one: All gate valves were closed, except one with the leak detector remained connected. After stationary conditions were maintained, the helium fluxes were measured, finally all pumping stations were compared. The idea is that the pumping station closest to the leak(s) should provide the highest helium level. Table II shows the helium fluxes for that measurement in arbitrary units, normalized to the helium background flux.

\begin{tabular}{|l|l|l|l|l|l|}
\hline & Station 11 & Station 21 & Station 31 & Station 50 & Station 51 \\
\hline $\begin{array}{l}\text { He-flux } \\
\text { (a.u.) }\end{array}$ & 882 & 912 & 885 & 878 & 886 \\
\hline
\end{tabular}

In this case, the helium flux for station no. 21 is outstanding (912 units) compared to the others (average value of the remaining four $=883$ units), possibly indicating the location of the leak(s) close to this station. Here, the station no. 50 remains unremarkable. 
So far, we have no good explanation why for the second method the station 21 shows no impact, and for the third method no. 50. We can only speculate and assume the following picture: due to the numerous in-vessel installations, the conductance between the leak(s) and the pumping stations will be different for the five pumping stations. In addition, helium will stick to surfaces due to adsorption. Assume further that the conductance between leak(s) and station no. 21 is comparably large: the helium from that local leak will be pumped away efficiently. In contrast, the conductance between leak(s) and station no. 50 is comparably small (compared to the other pumping stations): only a small fraction of the helium from the leak is pumped by station no. 50 , the rest is temporarily adsorbed by the numerous surfaces and/or pumped away by the other stations. For the second method this means that under normal conditions the helium is pumped away quickly by station no. 21. Closing the gate valve no. 21 will temporarily lead to an enhanced local adsorption in vicinity to no. 21, without large impact on the helium fluxes at the other locations, at least for the measurement period. The surfaces near location no. 50 are already saturated with helium. Closing the gate valve no. 50 will therefore promptly increase the helium flux to the other stations. For the third method this means that station no. 21 pumps away the highest flux of helium when open. The helium flux to station no. 50 is reduced because of the small conductance when open. If our speculation is correct, then the imbalances observed in the measured helium fluxes should disappear for long measurement periods (we guess in the range of several hours). However, due to the strict time schedule for the commissioning of W7-X, no confirmation could be done.

Finally, it should be mentioned that during each working day the helium background flux, as detected by the leak detector, showed slight and slow variations (in the range of $\approx 10-20 \%$ during 12 hours). They were presumably caused by slight temperature variations during the day in the experimental hall, and by all the other working activities around W7-X (welding, soldering, etc.), and finally of course by the inherent instability of the helium detector itself.

To conclude: a possible signature for leaks close to the pumping stations no. 21 and no. 50 is given from the time resolved measurement. However, contradictory indications result from the second and third method with the opened and closed gate valves. It can only be stated that the second method possibly indicates to a leak close to station no. 50 , the third method possibly to a leak close to station no. 21. This unsharp outcome might 
also be generated by the existence of more than only two leaks in vicinity to the affected pumping stations. Anyway: all indications taken together, towards leaks close to the locations no. 21 and 50, are strong enough to consider the attempt of a better localization by sniffer methods in the cryostat, when the vessel will be opened again. Albeit the sniffer method not as sensitive as the method applied so far, it will hopefully allow for a better localization of the leak(s).

When comparing all sub-systems in the W7-X cryostat, the leak search on the cryopiping system was considered as the most essential and critical. No access to a leak location is possible when the cryostat is cold, provisional closing of leaks is impossible. In addition, leaking helium will not be pumped away by the cold surfaces. Furthermore, leaks on cryo-pipes might increase in size when being cooled down, in an unpredictable manner. This makes them even more dangerous. In contrast to that: if air leaks on the outer cryostat skin occur during operation, the cold surfaces might act, at least provisionally, for a short time as cryo-pumps and will help stabilizing the pressure in the cryostat. Furthermore, one might have human access to the location of the air leak in order to repair it. Leaks between cryostat and plasma vessel will hardly be detectable during operation, because vacuum exists on both sides. If leaks in water pipes occur, one has, at least in principle, the possibility of making the risk consideration, and pump out the leaking pipe sections and leave certain components un-cooled for the time being, and one could try to avoid experimental scenario with an excessive power load to the components affected.

Fig. 19 shows the pressure development in the cryostat during the first 20 pumping days, after the major air leaks had been fixed. On the average, the pressure can be reduced each day in steps of about $0.5 \cdot 10^{-5} \mathrm{mbar}$, if all 5 pumping stations are utilized. The major gas constituent is now water vapor from out-gassing of the surfaces, with at least 99.8\% partial pressure compared to the total vacuum pressure. The partial pressure of helium, coming from the leaks in the cryo-pipes, is in the range $<10^{-7}$ mbar. 


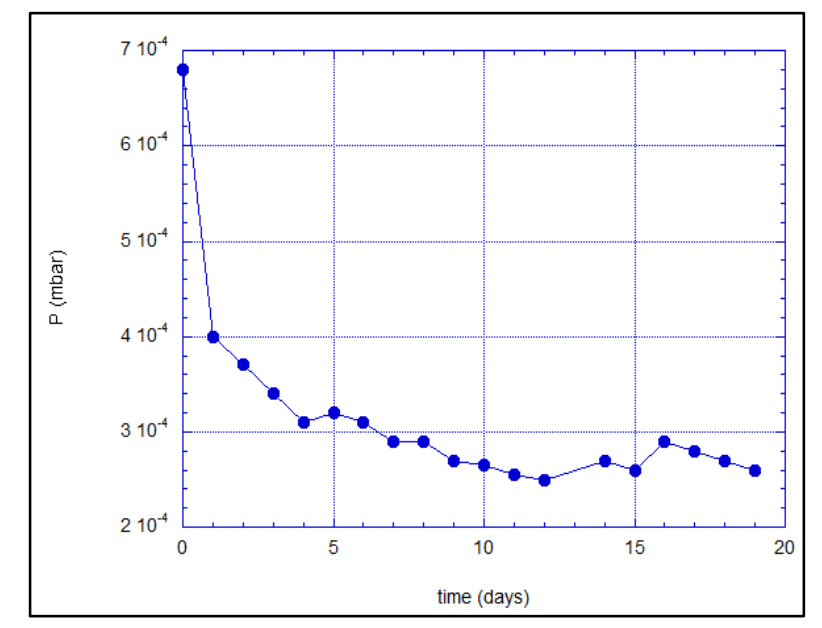

The total helium leak rate from the cryo-pipes was measured to $3 \cdot 10^{-5} \mathrm{mbar} \mathrm{l} / \mathrm{s}$ (see above) at a pressure of 4 bar in the tubes and at ambient temperature. This will be roughly the working pressure during operation. Taking into account the factor of 230 for the expansion of the supercritical helium, this might result in a total helium flux of $6.9 \cdot 10^{-3} \mathrm{mbar} \mathrm{l} / \mathrm{s}$, if the leaks don’t open further during cool-down. With a pumping speed of $500 \mathrm{l} / \mathrm{s}$, as measured for the five turbo pumping station, this results in a pressure increase of $1.4 \cdot 10^{-5}$ mbar. This is still low enough for a reliable superconductor operation. Because of the extremely tight time schedule for the start of the W7-X operation in summer 2015 [15], a leak repair in the cryostat is beyond scope, temporarily. The only measure that can be taken within these boundary conditions will be a partial opening of the chevrons between the pumping stations and the cryostat, thus enhancing the pumping speed considerably. The enhanced radiation power load to the cold components in the cryostat will remain acceptable, as calculations show. The extended leak search and (if possible) repair afterwards has to be shifted to the phase after the first experimental W7-X operation phase.

\section{Conclusions}

At the end of the commissioning phase of the cryostat vacuum system, the total gas flux from the cryostat was in the range of about $0.1 \mathrm{mbar}$ l/s, strongly dominated by the outgassing of water. In total, 28 leaks to the air could be detected on the outer cryostat skin and the inner plasma vessel, so far. At the beginning of the leak search, the integral leak rate was $\approx 100 \mathrm{mbar} 1 / \mathrm{s}$, at the end it was $\approx 4.6-9.6 \cdot 10^{-4} \mathrm{mbar} \mathrm{l} / \mathrm{s}$. The main reason for the leaks are always bad weld seams. Presumably two further leaks were found in the 
cryo-pipes with a leak rate of together $\approx 3 \cdot 10^{-5} \mathrm{mbar}$ l/s at 4 bar helium pressure. Only the water pipes in the cryostat were leak tight from the beginning.

The measured ion current ratio 18:28 by the mass spectrometer exceeded finally the value of 500, indicating that the remaining air leak rate is of the order of magnitude of some $10^{-4} \mathrm{mbar} \mathrm{l} / \mathrm{s}$. As a baking of the entire cryostat is not possible, it has to be relied on continuous pumping to get rid of the water. Purging with 50 mbar dry air to remove the water did not help, most likely because the applied air pressure was too small. The cryostat pressure was finally low enough that the decision could be taken to start the cool-down of the cryo-cooled components inside. When the temperature will drop, the surfaces should start pumping the water away, therefore a strong pressure reduction is expected to the range of about $10^{-6}$ mbar, such that finally the insulation vacuum will be good enough for the operation of the superconductors.

In total, 30 leaks could be detected within 4 months. Closing them by welding provided a cryostat that is appropriate to house the cold superconductors. Smaller leaks $\left(<10^{-4}\right.$ mbar l/s) might certainly still exist, however because they do not impede us from attaining an acceptable cryostat performance for the moment. Thus they will be subject to a future leak search and repair, when more time is available. If new cold leaks open, or existing leaks open even more during cool-down or the current operation, though, then this future leak search could become mandatory.

\section{Acknowledgements}

This work has been carried out within the framework of the EUROfusion Consortium and has received funding from the European Union's Horizon 2020 research and innovation programme under grant agreement number 633053. The views and opinions expressed herein do not necessarily reflect those of the European Commission.

\section{References}

[1] Beidler C, Harmeyer E, Herrnegger F, Kisslinger J, Igitkhanov Y, Wobig H. Fusion Technol. 1990; 17: 148-68. 
[2] Grieger G, Lotz W, Merkel P, Nührenberg J, Sapper J, Wobig H, et al. Phys. Fluids B 1992; 4: 2081-91.

[3] Klinger T, Baylard C, Beidler C, Boscary J, Bosch HS, Dinklage A, et al. Fusion Engineering and Design 2013; 88: 461-5.

[4] Spitzer L Phys. Fluids 1958; 1: 253.

[5] Rose DJ, Clark M. Plasmas and controlled fusion. MIT Press Cambridge, Mass. 1961.

[6] Reich J, Gardebrecht W, Hein B, Missal B, Starke F, Seidler KU. Fusion Engineering and Design 2003; 69: 345-8.

[7] Sapper J, W7-X Technical Group. Fusion Engineering and Design 1993; 20: 23-32.

[8] Hartfuss HJ, W7-X diagnostic team. Advanced diagnostics for magnetic and inertial fusion. Springer US, Kluwer Academic/Plenum Publishers, New York 2002; 371.

[9] Baldzuhn J, Ehmler H, Hoelting A, Sborchia C, Hertel K, Genini L, Schild T. IEEE Trans. Applied Superconductivity 2006; 16: 763.

[10] www.techneticsgroup.com/products/sealing-solutions/metal-seals/helicoflex/

[11] Baldzuhn J, Ehmler H, Genini L, Hertel K, Hoelting A, Sborchia C, Schild T. IEEE Trans. Applied Superconductivity 2008; 18: 509.

[12] Rademann D, W7-X Team. Symposium on Vacuum based Science and Technology, $6^{\text {th }}$ Annual DVG Meeting,, Greifswald, Germany, 2007.

[13] www.ilmvac.com

[14] www.itwwind.com 
[15] Sunn Pedersen T, W7-X Diagnostics Team. Bulletin of the American Physical Society, $56^{\text {th }}$ Annual Meeting of the APS Division of Plasma Physics, 2014; 59 (15): in press.

Figure captions

Fig. 1: Sketch of W7-X. A small part of the cryostat outer skin is shown (red, upper left), the superconducting coils (blue), the plasma vessel inside the coils, and the ports that provide access to the inner of the plasma vessel.

Fig. 2: CAD model of the W7-X cryostat. The cryostat outer skin is colored in green, the ports and openings are in blue. Some of the port openings are extended into the plasma vessel across the cryostat, some into the cryostat.

Fig. 3: Sketch of one of the cryostat pumping stations. At the valve "External access" additional components can be connected, as for instance a helium leak detector or a mass spectrometer.

Fig. 4: Scheme of the differential pumping arrangement for the mass spectrometer (QMS). Two possible inlets (arrows inward) can be connected to the pumping stations, shown in fig. 3. High inlet pressures can be reduced by two circular apertures with diameters of $0.3 \mathrm{~mm}$ and $0.1 \mathrm{~mm}$, respectively, to guarantee that the mass spectrometer inlet pressure never exceeds the allowed upper operation limit. This system can take mass spectra with inlet pressures between 1 bar and $10^{-7} \mathrm{mbar}$, depending on the combination of valves opened/closed.

Fig. 5: A collection of typical aluminum leak test chambers, together with the split silicone gaskets.

Fig. 6: Arrangement of a leak test sample, sitting in a local test chamber in the open $\mathrm{LN}_{2}$ bath.

Fig. 7: Time trace of the first complete pump-down of the W7-X cryostat. At a pressure below 100 mbar, the roots pumps were switched on. 
Fig. 8: Close-up photograph of one of the critical T-shaped weld seams

Fig. 9: Mass spectrum of the residual gas during the initial pump-down, showing the atomic mass composition in the cryostat.

Fig. 10: Typical time traces of the helium leak rate as measured during the helium puffing leak search. The red arrows indicate moments when helium was puffed in close vicinity to a leak in the cryostat. The y-axis gives the measured He-Flux, however not yet absolutely calibrated, because only a partial flux was measured when being connected to the pumping station. The absolute leak rate is estimated to be larger by roughly a factor of $10^{5}$. Note the long time decay after the helium puff back to the previous background level. After a leak was successfully detected, typically several 10 minutes waiting time were required until the helium signal was stable enough to continue the leak search.

Fig. 11: Mass spectrum, taken directly after the major leaks had been closed. Now the signatures of the air peaks disappeared almost completely, and water peaks (masses 18, $17,16)$ dominate. The ratio of the ion currents for the masses 18:28 is here 80 and tends to increase further. After a few weeks of pumping time, the ratio increased up to $\approx 400$.

Fig. 12: Time traces of the ion currents measured by the mass spectrometer for 18 amu/e (water) and $28 \mathrm{amu} / \mathrm{e}$ (nitrogen), during the three phases I - III.

Fig. 13: Time traces of the mass spectrometer ion currents for several masses that could be detected as background in the cryostat. The vertical bars denote moments in time, when a new water circuit was filled with neon. The entire measurement period is about 6 hours long. At the beginning, obviously some out-gassing of the filament perturbs the measurement.

Fig. 14: CAD model of one fifth of the W7-X plasma vacuum vessel. The surrounding cryostat and the coils are not shown. The ports connect the plasma vessel inner side with the exterior. Each port has $1-2$ bellows. Some of them are marked by yellow ellipses.

Fig. 15: A part of the helium cryo-pipes in the cryostat for modules 1-3 (M1 - M3). Shown are some of the supply and exhaust lines for the coil winding packs (black rectangles) and the current leads (CL). 
Fig. 16: Time traces of the helium detector signal, showing the temporal increase of the helium flux after application of pressure steps to the three ring lines. The helium background for the test of "L1" was slightly higher than for the other two due to the tests done before. The time response of line “L2N2” was the fastest. That measurement was taken on pumping station 50 . The vertical line at $\mathrm{t}=295 \mathrm{~s}$ shows the opening moment of the corresponding inlet valve.

Fig. 17: Delay time for the increase of the helium flux on the leak detector versus the number of the pumping station. Two test runs are shown, one with slightly higher pressure (dots, solid line) and one with slightly lower pressure (squares, dashed line). The differences between the two measurements indicate the typical systematic errors associated with this method.

Fig. 18: Time traces of the helium leak detector, when being connected to pumping station 11. The four maxima were recorded when the pumping stations with the corresponding numbers were closed. A slightly higher helium flux was observed when station 50 was closed (black ellipse), compared to the others. The (slow) helium background drift is indicated as horizontal line on the top. A constant helium pressure of 4 bar is applied to the mesh all the time.

Fig. 19: Cryostat pressure time curves, after the major leaks had been repaired.

Table captions

Table I: Relative changes of the helium fluxes in the leak detector.

Table II: Normalized helium fluxes in arbitrary units, if only one pumping station is connected to the cryostat, with constantly 4 bar on the mesh for all 5 measurements. The statistical noise is in the range of \pm 1 unit. 